\title{
Philosophiques
}

\section{Réplique à Pierre Raymond}

\section{Yvon Gauthier}

Volume 5, numéro 1, avril 1978

URI : https://id.erudit.org/iderudit/203094ar

DOI : https://doi.org/10.7202/203094ar

Aller au sommaire du numéro

Éditeur(s)

Société de philosophie du Québec

ISSN

0316-2923 (imprimé)

1492-1391 (numérique)

Découvrir la revue

Citer ce document

Gauthier, Y. (1978). Réplique à Pierre Raymond. Philosophiques, 5(1), 192-194.

https://doi.org/10.7202/203094ar

Ce document est protégé par la loi sur le droit d'auteur. L'utilisation des services d'Érudit (y compris la reproduction) est assujettie à sa politique d'utilisation que vous pouvez consulter en ligne.

https://apropos.erudit.org/fr/usagers/politique-dutilisation/
Cet article est diffusé et préservé par Érudit.

Érudit est un consortium interuniversitaire sans but lucratif composé de l’Université de Montréal, l’Université Laval et l’Université du Québec à Montréal. Il a pour mission la promotion et la valorisation de la recherche. https://www.erudit.org/fr/ 


\section{RÉPLIQUE À PIERRE RAYMOND}

par Yvon Gauthier

Monsieur Pierre Raymond

École Normale Supérieure

45, rue d'Ulm

75230 Paris

Cher monsieur Raymond,

Je viens de recevoir votre texte, qui m'est parvenu par la bande, puisqu'il ne m'était pas destiné, semble-t-il. Vos réactions, que vous annoncez vives, me paraissent bien anodines. J'étais disposé à reconnaître une certaine virulence gratuite dans quelques passages de mon article qui vous concernent, mais votre acrimonie vindicative (je mets à part les quelques insultes inoffensives) $m$ 'incite à vous répondre en staccato, comme il convient dans l'art mineur de la réplique (que vous ne semblez pas savoir manier avec élégance).

1. Fondements-fondation : il est vrai que Cavaillès a suggéré ce thème dans sa thèse de doctorat — j'ai indiqué ailleurs les failles des surinterprétations de Cavaillès. Mais ce thème devient chez vous un leitmotiv, un motto qui n'est jamais une reprise critique, mais un babil répétitif. J'ai bien dit que la philosophie mathématique de Cavaillès pouvait encore inspirer le philosophe instruit du savoir actuel.

2. Absurdités de tous les jours : l'article de Sinaceur, auquel vous faites allusion, est généralement bien informé. La phrase que je cite de vous est bien de votre cru et non du meilleur, c'est l'interprétation que vous avez cru pouvoir tirer du court exposé de Sinaceur. Ce n'est pas une citation sauvage, comme dans de nombreux autres cas.

3. Discours sur les discours ... : la thèse que vous annonciez, i.e. les mathématiques comme expérimentation, se résume au 
commentaire d'articles de vulgarisation ou de travaux de philosophes.

4. Philosophe, logicien : vous accordez beaucoup d'importance aux étiquettes. Pour ma part, je renonce allégrement à celle de philosophe, s'il faut faire la philosophie à votre manière.

5. Marxiste comme tout le monde : l'ironie de la formule vous a échappé ; il est vrai que c'est une "gauthièreté ".

6. Dialogue : les passages qui vous concernent sont repris d'un compte rendu dans Dialogue. Je n'ai pas cru bon d'y ajouter quoi que ce soit et de vous accabler davantage deux ans après. Si je n'ai pas cité dans un autre contexte votre participation à Philosopbie et Calcul de l'infini, c'est qu'elle se réduit à un fouillis philosophico-historique sans aucun rapport avec les exposés mathématiques de l'ouvrage. Si, par ailleurs, j'ai éliminé la dernière phrase publicitaire, c'est que je n'étais plus sûr après vous avoir lu de nouveau que " matérialisme " était encore une bonne marque de commerce. Vous conviendrez, qu'avec les deux maigres pages que je vous consacre dans mon article, je n'ai pas voulu faire ma publicité sur votre dos trop fragile.

7. Du nouveau : je n'avais cependant pas lu votre dernier travail Logique et matérialisme dialectique. Je commence par en dire du bien. Je suis d'accord avec l'une de vos thèses principales sur le matérialisme dialectique : il n'y a plus de matérialisme dialectique, métaphysique anachronique d'un nom inassignable, la matière. Badiou, dans sa "Théorie de la contradiction " est bien en deçà. Malheureusement, il n'y a pas que cette perspective progressiste. Ce que vous dites sur la logique et sur la philosophie positiviste me fait regretter encore une fois que vous abordiez ces problèmes. Je n'en veux ici qu'un seul exemple : vous commentez l'énoncé de Russell (p. 142-143) : "une proposition vraie est impliquée par n’importe quelle proposition " en disant qu'il faut ajouter "supposée vraie ". N'importe quel étudiant de logique élémentaire sait que cet axiome médiéval, "Tout énoncé vrai suit de n'importe quel énoncé " (y compris sa propre négation, "consequentia mirabilis") est le pendant de l'axiome " Ex falso sequitur quodlibet ». Russell, évidemment, savait tout cela. 
8. Quant aux cautions mathématiques, j'ai toutes celles qu'il me faut (en particulier, le seul mathématicien directement concerné m'a déjà répondu, à ma plus grande satisfaction).

9. Je n'ai plus la patience de commenter "Interdictions philosophiques". Ce sont des généralités sans poids.

10. J'espère que ce n'est là que le début d'un échange fructueux. Vous avez sans doute au Québec quelques lecteurs, plus patients et plus innocents. Nos querelles ne sont qu'académiques, nous sommes unis dans un même combat, au fond. Je vous invite quand même à lire mon ouvrage (que je vous envoie sous pli séparé) ou mes ouvrages ; ils ne sont pas sans défauts, mais il y a là un discours apparemment cohérent ; on peut commencer par là.

Avec mes salutations cordiales,

Yvon Gauthier, professeur titulaire, Université de Montréal 